\title{
CONTINUOUS OR DISCRETE? A MORPHOSPACE COMPARISON OF LIVING AND FOSSIL GLOBOROTALIA
}

\author{
TABACHNICK*, R. Elena, DePaul University, 243 S. Wabash Ave., Chicago, IL \\ 60604, U.S.A.
}

One interesting attribute of planktonic foraminifera is the extent to which intermediates exist between morphologically defined species. This continuity can be found between ancestral and descendant morphs, in geographical clines across faunal provinces and between sympatric morphospecies. In some Miocene populations of Globorotalia, individuals intermediate between morphospecies are as abundant as individuals that can be placed into morphospecies. These foraminifera don't seem to "come in species" at all.

Living Globorotalia from 30 plankton tow samples do exhibit variation suggestive of discrete morphospecies. In samples ranging across several major faunal provinces of the North Atlantic, most individuals can be qualitatively sorted into distinct morphological groups. In many samples the variation suggests the presence of a single morphospecies. Others seem to contain two or more distinct morphospecies. In samples with the highest diversity, primarily from the equatorial regions, there may be gradation between two or more of these morphospecies, but such "species groups" can still be distinguished from others in the same sample.

What does it mean to say there is continuity or discrete variation in a morphological distribution? Can this difference be quantified? Can it be analyzed as features of distributions within a morphospace? Comparison of the distributions of Miocene and living Globorotalia may provide insight into these questions. If such a distributional difference can be ascertained, then we are free to explore likely explanations. For example, continuity between morphospecies may result from averaging ecophenotypic variation present in living populations over the time and geographic range represented by fossil populations. Alternatively, planktonic foraminifera may form discrete morphospecies at some times in their evolutionary history but not at others. This difference could be part of the general, foraminiferan evolutionary cycle of diversification followed by diversity reduction. Or possibly, the planktonic foraminifera may form species facultatively. 